1 SPARC: a method to genetically manipulate precise proportions of cells.

2 Jesse Isaacman-Beck ${ }^{1}$, Kristine C. Paik ${ }^{1,2}$, Carl F. R. Wienecke ${ }^{1}$, Helen H. Yang ${ }^{3}$,

3 Yvette E. Fisher ${ }^{3}$, Irving E. Wang ${ }^{1,4}$, Itzel G. Ishida ${ }^{5}$, Gaby Maimon ${ }^{5}$, Rachel I. Wilson ${ }^{3}$,

4 Thomas R. Clandinin ${ }^{1^{*}}$

5

6 AFFILIATIONS

$7 \quad{ }^{1}$ Department of Neurobiology, Stanford University, Stanford, CA 94305

8 2Present address: Department of Medicine, Weill Cornell Medical College, New York,

$9 \quad$ NY 10065

$10{ }^{3}$ Department of Neurobiology and Howard Hughes Medical Institute, Harvard Medical

11 School, Boston, MA 02115

$12 \quad{ }^{4}$ Present address: Freenome, South San Francisco, CA 94080

$13{ }^{5}$ Laboratory of Integrative Brain Function and Howard Hughes Medical Institute, The

14 Rockefeller University, New York, NY 10065

15 *Correspondence: trc@stanford.edu

\title{
17 Abstract
}

18 Many experimental approaches rely on controlling gene expression in select subsets of

19 cells within an individual animal. However, reproducibly targeting transgene expression

20 to specific fractions of a genetically-defined cell-type is challenging. We developed

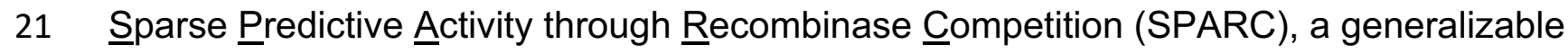

22 toolkit that can express any effector in precise proportions of post-mitotic cells in

23 Drosophila. Using this approach, we demonstrate targeted expression of many 
24 effectors and apply these tools to calcium imaging of individual neurons and optogenetic

25 manipulation of sparse cell populations in vivo.

\section{Main Text}

Genetic labeling and manipulation of small groups of cells in somatic mosaic

28 animals have provided significant insights into many aspects of biology and have been

29 particularly impactful in studies of the nervous system. Indeed, measurement and

30 manipulation of genetically defined cell types has become central to neural circuit

31 dissection in many systems ${ }^{1}$. Especially powerful are paradigms in which one measures

32 the phenotypes of stochastically selected individual cells within otherwise

33 unmanipulated populations. However, few methods exist for selectively manipulating a

34 desired fraction of cells of the same genetically-defined type. In rodents, sequential

35 recombinase-mediated switches can label subpopulations of neurons, but require labor-

36 intensive titration of viruses ${ }^{2}$. In Drosophila, GAL4 and split GAL4 lines enable targeting

37 of single cell types ${ }^{3}$, but selective manipulation of subsets of neurons of the same type

38 remains challenging. For example, effector expression can be restricted by limiting the

39 spatial and/or temporal expression of a recombinase, but this necessitates user-

40 dependent heat shock or chemical induction, and in some cases, cannot be used in

41 post-mitotic cells ${ }^{4-6}$. Therefore, a routine all-genetic method of expressing effectors in

42 defined fractions of post-mitotic cells of the same type would provide a powerful means

43 of dissecting cellular and genetic functions.

To address this need, we developed SPARC, a toolkit to express any effector in

45 precise proportions of post-mitotic cells labelled by the GAL4-UAS system (Fig. 1A, S1, 
S2) ${ }^{7}$. The core of this toolkit is a set of bistable UAS-driven constructs that can be

47 switched on or off in different relative proportions of cells, depending on their sequences. We designed each UAS-driven construct, so that PhiC31 recombinase ${ }^{8}$

49 could irreversibly recombine one of two competing attP target sequences with one attB target sequence. The reaction mediated by the first attP would remove a stop cassette

51 to enable effector expression in cells expressing Gal4, while the reaction using the

52 second attP would leave this stop intact and prevent expression (Fig. S1). Truncating

53 canonical attP sequences diminishes the efficacy of recombination in vitro ${ }^{9}$. Based on

54 this, we reasoned that truncating the first attP relative to the second would shift the

55 equilibrium to favor retention of the stop cassette and result in sparser effector

56 expression in vivo.

To test this concept, we generated plasmids and transgenic flies bearing SPARC

58 constructs expressing the calcium indicator GCaMP $6 \mathrm{f}^{10}$ and including one of three

59 different attP variants at the first position (canonical: attP60; truncated: attp38 or attp34) $)^{9}$. We also generated transgenic flies that express PhiC31 recombinase in all

61 post-mitotic neurons under the control of the synaptobrevin promoter ${ }^{3}$. We then drove

62 expression of each SPARC construct in one of the largest genetically-defined

63 populations of neurons in the Drosophila optic lobe, T4 and T5 cells ${ }^{11}$, in animals that

64 express PhiC31 pan-neuronally. Across SPARC-GCaMP6f variants, we observed

65 progressively fewer labeled neurons (Fig. 1C-E). SPARC-attP60-GCaMP6f labeled

66 many overlapping neurons, SPARC-attP38-GCaMP6f labeled an intermediate number

67 of neurons and SPARC-attP34-GCaMP6f labeled individual neurons whose dendrites

68 could be visualized (Fig 1E inset). We therefore named these variants SPARC-D 
69 (Dense), SPARC-I (Intermediate), and SPARC-S (Sparse). To determine the

70 percentage of these neurons labeled by each SPARC module, we expressed

71 myristoylated-tdTomato (myr-tdT) in all T4 and T5 neurons in parallel with SPARC-

72 GCaMP6f and counted singly and doubly labeled T4 and T5 cell bodies (Fig. 1F and

73 S3). For SPARC-D-GCaMP6f we observed effector expression in $\sim 45 \%$ of T4 and T5

74 neurons. In comparison, SPARC-I-GCaMP6f labeled $\sim 12 \%$ of T4 and T5 neurons, while

75 SPARC-S-GCaMP6f labeled only $\sim 4 \%$ of these neurons. We observed similar results in

76 Kenyon cells, Lobula-Columnar neurons, and several columnar neurons in the optic

77 lobe (Fig. S4 and data not shown). These data demonstrate that the SPARC module

78 can reproducibly determine the fraction of cells that express effector over a more than

79 10-fold range across cells and animals. As SPARC-S can readily label T4, T5 and

80 Kenyon cells, very common cell types in the fly brain, these studies argue that one of

81 the three SPARC modules should allow targeting of individual cells of any cell type of

82 interest.

83 To generalize this technique, we next made SPARC-LexA::p65 transgenes.

84 LexA::p65 is a transcription factor that drives expression of transgenes under the control

85 of the lexAop promoter ${ }^{12}$; this system is orthogonal to the UAS-GAL4 expression

86 system. We expressed SPARC-LexA::p65 in Mi1 neurons of the Drosophila optic lobe

87 (Fig 1B), and found that in the absence of PhiC31 recombinase, SPARC-LexA::p65

88 labels $100 \%$ of these neurons with lexAop-myr-tdT (Fig. 1G-G"). This result suggested

89 that the widely-used stop cassette ${ }^{13}$ that we used in the initial SPARC design might

90 permit a low level of read-through that can be detected by sensitive outputs like

91 LexA::p65 (or mCD8::GFP, data not shown). 
To avoid this read-through, we generated SPARC2, in which we incorporated two

93 self-cleaving ribozymes from the Hepatitis Delta Virus (HDV) into the SPARC module

94 (Fig. 1A). We reasoned that these self-cleaving ribozymes should truncate any read-

95 through transcript prior to translation ${ }^{14,15}$. We first examined SPARC2-LexA::p65

96 transgenes in Mi1 neurons in the absence of PhiC31 and observed a 10,000-fold

97 decrease in read-through ( $0.01 \%$ of Mi1 labeled with lexAop-myr-tdT, Fig. $\left.1 \mathrm{H}-\mathrm{H}^{\prime \prime}\right)$.

98 Importantly, the D, I, and S variants of SPARC2-LexA::p65 behaved similarly to

99 SPARC-GCaMP6f transgenes (Fig. 1I-K"). Also quantitative measurement of SPARC2-

100 mCD8::GFP in Mi1 cells showed the same three levels of expression(Figure S4I-L).

101 Thus, HDV ribozymes effectively eliminate read-through and enable SPARC2

102 transgenes to express both direct and amplifying effectors in three different proportions

103 of cells.

104 Next, to investigate the functional utility of SPARC, we first used SPARC-S-

105 GCaMP6f to image calcium $\left(\mathrm{Ca}^{2+}\right)$ response in the dendrites of individual T5 neurons.

106 These neurons preferentially respond to visual motion in one direction, a direction

107 selectivity that is first observed in their dendrites ${ }^{16}$. Previous attempts to image from

108 individual T5 cells relied on laborious FlpOut approaches that required titrated and

109 temporally precise heat shocks of Drosophila larvae to restrict effector expression to a

110 subset of cells ${ }^{16-18}$. In contrast, the SPARC method consistently labeled fewer T5

111 neurons, and labeled them more sparsely, than the sparsest FlpOut labeling using brief

112 and developmentally late heat-shock (Fig. 1E, 2A,B). More importantly, when we

113 imaged visually-evoked $\mathrm{Ca}^{2+}$ responses in regions of interest (ROIs) representing T5

114 dendrites, we observed that the fluorescent signals from SPARC-labeled ROls were 
115 significantly more direction selective than those from FlpOut-labeled ROls (DSI; Fig. 2C-

116 E). This result reflects the fact that SPARC labeling was sparser than the sparsest

117 FlpOut labeling we could achieve. As a consequence, SPARC ROIs more nearly

118 captured signals from single cells, while FlpOut ROls likely included signals from

119 multiple labeled cells with different directional preferences (see supplemental methods).

120 Thus, both anatomical and functional evidence suggests that SPARC better isolates

121 single T5 dendrites more easily and more consistently than standard FIpOut

122 approaches.

123 Then, to determine if we could use this approach to manipulate the activity of

124 neuronal subpopulations, we generated SPARC2-CsChrimson::tdTomato ${ }^{19}$ transgenic

125 flies. We expressed this construct in Ring (R) neurons, GABAergic neurons that send

126 sensory input to the central complex ${ }^{20}$. R neurons are divided into types based on

127 morphology ${ }^{21}$, here we focused on the R2 type. We expressed SPARC2-D-

128 CsChrimson::tdTomato in a subset of R2 neurons (Figures 2F-H”). We observed that

129 tdTomato ${ }^{+}$R2 neurons were depolarized by light, while tdTomato- R2 neurons were not

130 depolarized (Figure 2I, J). Indeed, tdTomato- R2 neurons were slightly hyperpolarized

131 by light (Figure 2I, J), implying that these R2 neurons were postsynaptic to other R2

132 neurons that express CsChrimson. Thus, the SPARC system enables optogenetic

133 activation of sparse cell populations within a single cell type, allowing us to discover

134 evidence for mutual inhibitory interactions within a cell type. Although it was already

135 known that some $\mathrm{R}$ neuron types inhibit other $\mathrm{R}$ types ${ }^{20}$, it was not previously known

136 that there is mutual inhibition between $\mathrm{R}$ neurons of the same type. 
The SPARC and SPARC2 toolkit includes direct effector transgenes that can be used to label cells (mCD8::GFP), to observe changes in intracellular calcium concentration (GCaMP6f, jGCaMP7f) and membrane potential (ASAP2f), as well as to

140 optogenetically modulate neuronal activity (CsChrimson). In addition, the availability of

141 the indirect effector transgene SPARC2-LexA::p65 opens the possibility of sparsely

142 expressing a large range of additional existing effectors under the control of lexAop

143 (Figure S5). To provide the flexibility to target both neuronal and non-neuronal cells, we

144 also generated transgenic animals that express PhiC31 pan-neuronally (nSyb-PhiC31),

145 ubiquitously (tub-PhiC31) and in any cell type labeled by GAL4 (UAS-PhiC31). Thus, by

146 simply generating flies with the appropriate combination of transgenes (Fig. S6), one

147 can perform a diverse array of experiments on single cells or precise proportions of cells

148 of a given genetically defined type. Moreover, each element of this toolkit is modular,

149 allowing users to easily incorporate any current or future genetically encoded effector

150 (Fig. S3). In the context of the nervous system, SPARC, SPARC2 and future variations

151 will allow convenient and unparalleled access to define the heterogeneity of single

152 neuron contributions to neural circuit processing. In non-neuronal cells, SPARC will

153 enable wide-ranging studies that exploit mosaic analysis to investigate the cell biology

154 and physiology of post-mitotic cells. Finally, as PhiC31 functions in both the mouse and 155 fish 22,23 , we anticipate that this strategy will be widely generalizable to other model 156 systems.

158 Acknowledgements: We thank members of the Clandinin, Wilson, and Maimon labs

159 for discussion of the project and manuscript. We thank Arun Chakravorty for generating 
160 the SPARC-jGCaMP7f plasmids, Scott Gratz, Kate O’Connor-Giles, ChiChi Xie, Liqun

161 Luo, Barrett Pfeiffer and David Anderson for providing template plasmids for molecular

162 cloning. We also thank Gerald Rubin and Heather Dionne for sharing split-Gal4 stocks,

163 Norbert Perrimon for sharing Cas9 stocks, and note that stocks obtained from the

164 Bloomington Drosophila Stock Center (NIH P40OD018537) were used in this study. The

165 project was supported by the NIH (R01EY022638 and 5P30EY026877 to T.R.C.,

166 5U19NS104655 to T.R.C. and R.E.W. J.I-B. is an Arnold O. Beckman Postdoctoral

167 Fellow, H.H.Y. is an HHMI fellow of the Jane Coffin Childs Memorial Fund for Medical

168 Research, Y.E.F. is supported by a Hanna H. Grey Fellowship from HHMI, C.F.R.W. is

169 supported by an NSF Graduate Research Fellowship (DGE - 1656518). R.I.W. and

170 G.M. are HHMI investigators.

171

172 Author contributions: J.I-B., H.H.Y, I.E.W. and T.R.C conceived the study. J.I-B,

173 C.F.R.W., H.H.Y., and Y.E.F. designed and performed the experiments under the

174 supervision of T.R.C. and R.I.W. J.I-B., K.C.P., H.H.Y., Y.E.F., and I.G.I generated,

175 maintained, and/or validated transgenic fly stocks under the supervision of T.R.C,

176 R.I.W., and G.M. J.I-B., C.F.R.W., H.H.Y., Y.E.F. and K.C.P. analyzed the data. J.I-B.

177 and T.R.C. prepared the manuscript with contributions from C.F.R.W., H.H.Y., and

178 Y.E.F.

179

180 Competing Interests statement: The authors declare no competing financial interests.

182 Methods: Attached as Supplemental Methods. 


\section{References:}

184 1. Luo, L., Callaway, E. M. \& Svoboda, K. Genetic Dissection of Neural Circuits: A 185 Decade of Progress. Neuron 98, 256-281 (2018).

$1862 . \quad$ Lin, R. et al. Cell-type-specific and projection-specific brain- wide reconstruction of single neurons. Nat. Methods 1-9 (2018). doi:10.1038/s41592-018-0184-y

3. Jenett, A. et al. A GAL4-driver line resource for Drosophila neurobiology. CellReports 2, 991-1001 (2012).

4. Lee, T. \& Luo, L. Mosaic analysis with a repressible cell marker for studies of gene function in neuronal morphogenesis. Neuron 22, 451-461 (1999).

5. Del-Valle-Rodriguez, A., Didiano, D. \& Desplan, C. Power tools for gene expression and clonal analysis in Drosophila. Nat. Methods 9, 47-55 (2011).

6. Sethi, S. \& Wang, J. W. A versatile genetic tool for post-translational control of gene expression inDrosophila melanogaster. eLife 6, e04577 (2017).

7. Brand, A. H. \& Perrimon, N. Targeted gene expression as a means of altering cell fates and generating dominant phenotypes. Development 118, 401-415 (1993).

8. Groth, A. C., Olivares, E. C., Thyagarajan, B. \& Calos, M. P. A phage integrase directs efficient site-specific integration in human cells. Proc. Natl. Acad. Sci. U.S.A. 97, 5995-6000 (2000).

9. Groth, A. C., Olivares, E. C., Thyagarajan, B. \& Calos, M. P. A phage integrase directs efficient site-specific integration in human cells. Proc. Natl. Acad. Sci. U.S.A. 97, 5995-6000 (2000).

10. Chen, T.-W. et al. Ultrasensitive fluorescent proteins for imaging neuronal activity. Nature 499, 295-300 (2013). 
11. Pinto-Teixeira, F. et al. Development of Concurrent Retinotopic Maps in the Fly

Motion Detection Circuit. Cell 1-26 (2018). doi:10.1016/j.cell.2018.02.053

12. Lai, S.-L. \& Lee, T. Genetic mosaic with dual binary transcriptional systems in

Drosophila. Nat Neurosci 9, 703-709 (2006).

13. Pfeiffer, B. D. et al. Refinement of Tools for Targeted Gene Expression in

Drosophila. Genetics 186, 735-755 (2010).

212 14. Perrotta, A. T. \& Been, M. D. A pseudoknot-like structure required for efficient self-cleavage of hepatitis delta virus RNA. Nature 350, 434-436 (1991).

214 15. Wernet, M. F., Klovstad, M. \& Clandinin, T. R. A Drosophila toolkit for the visualization and quantification of viral replication launched from transgenic genomes. PLoS ONE 9, e112092 (2014).

217 16. Fisher, Y. E., Silies, M. \& Clandinin, T. R. Orientation Selectivity Sharpens Motion

Detection in Drosophila. Neuron 88, 390-402 (2015).

219 17. Leong, J. C. S., Esch, J. J., Poole, B., Ganguli, S. \& Clandinin, T. R. Direction

Null-Direction Suppression. Journal of Neuroscience 36, 8078-8092 (2016).

222 18. Wienecke, C. F. R., Leong, J. C. S. \& Clandinin, T. R. Linear Summation

225 19. Klapoetke, N. C. et al. Independent optical excitation of distinct neural 
227 20. Xie, X. et al. The laminar organization of the Drosophila ellipsoid body is semaphorin-dependent and prevents the formation of ectopic synaptic connections. eLife 6, e04577 (2017).

230 21. Omoto, J. J. et al. Neuronal Constituents and Putative Interactions Within the 231 Drosophila Ellipsoid Body Neuropil. Front. Neural Circuits 12, 103 (2018).

232 22. Mosimann, C. et al. Site-directed zebrafish transgenesis into single landing sites with the phiC31 integrase system. Dev. Dyn. 242, 949-963 (2013).

234 23. Olivares, E. C. et al. Site-specific genomic integration produces therapeutic Factor IX levels in mice. Nature Biotechnology 20, 1124-1128 (2002).

236 24. Wystrach, A., Dewar, A. D. M. \& Graham, P. Insect Vision: Emergence of Pattern 237 Recognition from Coarse Encoding. Current Biology 24, R78-R80 (2014). 
bioRxiv preprint doi: https://doi.org/10.1101/788679; this version posted September 30, 2019. The copyright holder for this preprint (which was not certified by peer review) is the author/funder, who has granted bioRxiv a license to display the preprint in perpetuity. It is made available under aCC-BY-NC-ND 4.0 International license.

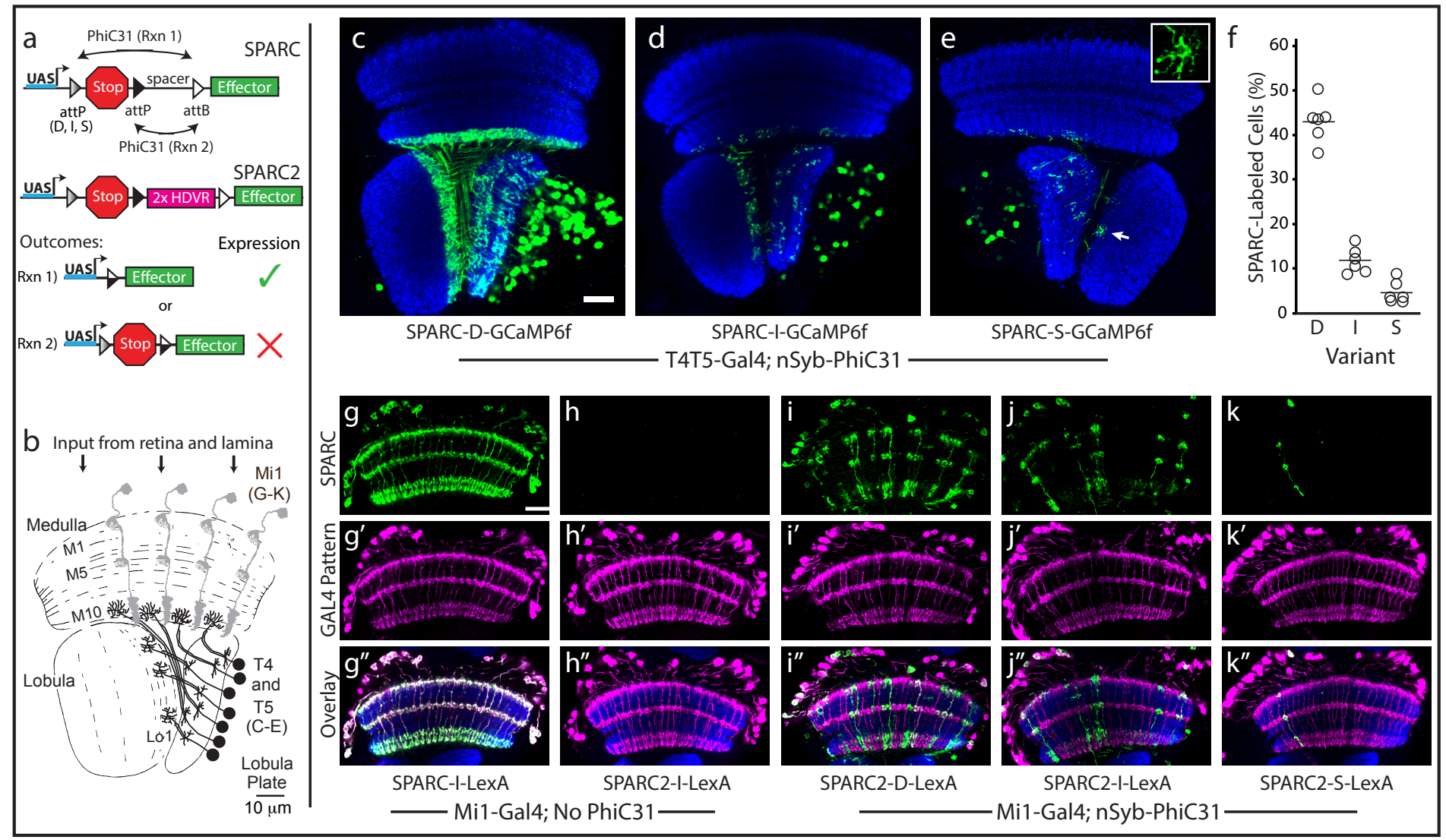

Figure 1: SPARC and SPARC2 enable predictive expression of effectors at three levels. (a) Schematic of SPARC and SPARC2 modules. PhiC31 recombines one of two competing attP target sequences with one attB target sequence. Truncating the first attP progressively favors retention of the stop cassette, preventing expression (Dense-60bp, canonical sequence; Intermediate - 38bp; Sparse - 34bp). SPARC2 incorporates a 2 X Hepatitis Delta Virus Ribozyme (HDVR) sequence to prevent read-through in the absence of PhiC31. (b) Schematic of the Drosophila optic lobe highlighting T4, T5 and Mi1. (c-e) GCaMP6f expression (green) in T4 and T5 neurons counterstained with anti-Bruchpilot (blue). (c) SPARC-D-GCaMP6f (d) SPARC-I-GCaMP6f $($ (e) SPARC-S-GCaMP6f, arrow points to dendrite shown in inset (f) Percentage of T4 and T5 neurons labeled by different SPARC modules (see Fig. S3). N = 6 optic lobes and $>400$ cell bodies/genotype. (g-k) LexA::p65-driven expression of lexAop-myr-tDT (green, g-k), in Mi1 neurons (magenta, $g^{\prime}-k^{\prime}$ ) counterstained with anti-Bruchpilot (blue, overlay, g"-k"). (g) SPARC-I-LexA::p65 no PhiC31. LexA::p65 is expressed in all Mi1 neurons in the absence of PhiC31 in these animals. (h) SPARC2-I-LexA::p65 no PhiC31. LexA::p65 is not expressed in Mi1 neurons in the absence of PhiC31, consistent with an absence of read-through. (i) SPARC2-D-LexA::p65. (j) SPARC2-I-LexA::p65 (k) SPARC2-S-LexA::p65. Scale bars, $10 \mu \mathrm{m} ; \mathrm{N}>10$ optic lobes per condition. 

under aCC-BY-NC-ND 4.0 International license.

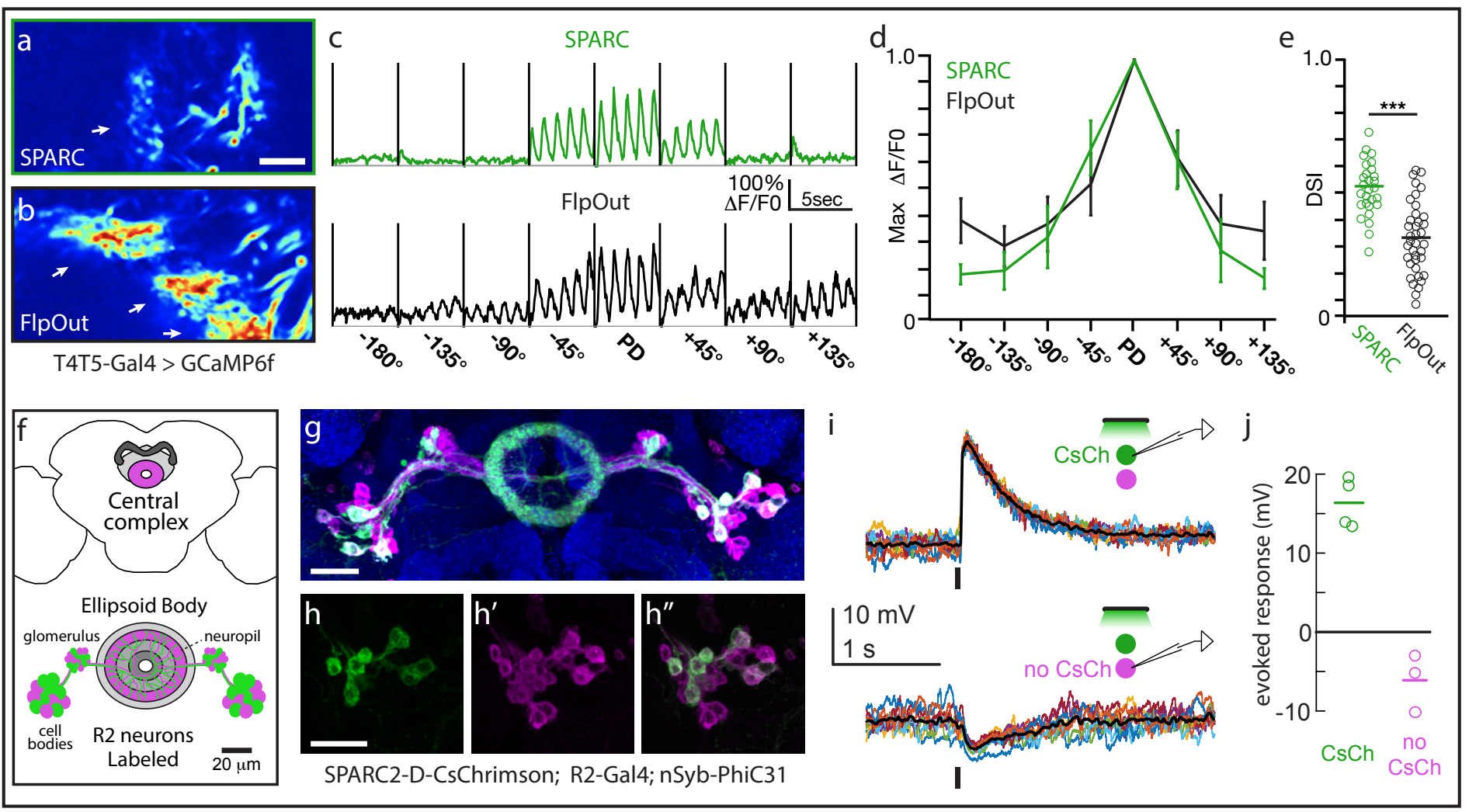

Figure 2: SPARC and SPARC2 enable calcium imaging of single neurons and optogenetic stimulation of sparse cell populations. (a, b) Normalized averaged fluorescence intensity of GCaMP6f in T5 dendrites sparsely labeled using (a) SPARC-S-GCaMP6f or (b) FlpOut-GCaMP6f. Arrows point to dendrites. (c-d) GCaMP6f fluorescence responses ( $\triangle \mathrm{F} / \mathrm{F0}$ ) of T5 dendritic ROls to sinusoidal gratings moving in one of eight different directions. PD denotes the preferred direction of each cell with the angular deviation from PD in degrees (c) Averaged responses of a representative ROI labeled using SPARC-S-GCaMP6f (green) or FlpOut-GCaMP6f (black). (d) Normalized tuning curves averaged across all T5 dendritic ROls labeled by SPARC-S-GCaMP6f (green) or FlpOut-GCaMP6f (black) (e) Direction selectivity indices (DSI) for each T5 dendritic ROI labeled by SPARC-S-GCaMP6f or FlpOut-GCaMP6f, $n=8$ flies and 37 units per condition; ${ }^{* * *} \mathrm{p}<0.001$. (f) Schematic of Central Complex and Ellipsoid Body depicting SPARC2-labeled R2 Ring neurons (adapted from24). (g) SPARC2-D-CsChrimson::tdTomato-3.1 expression (tdTomato; green) in R2 Ring neurons (mCD8::GFP; magenta) counterstained with anti-Bruchpilot (blue). (h-h") Closeup of cell bodies in (g) showing (h) CsChrimson expression in ( $h^{\prime}$ ) R2 cells. ( $h^{\prime \prime}$ ) Overlay. (I) Current clamp recordings of single tdTomato+ (top) and tdTomato- (bottom) R2 neurons. Stimulus is a 50 ms pulse of green light; 10 trials each (colored lines), mean response (black line). (j) Average evoked response (open circles) and mean population response (line) of R2 neurons, both tdTomato+ (green, $\mathrm{N}=4$ ) and tdTomato(magenta, $N=3)$. Scale bars: $10 \mu \mathrm{m}(\mathrm{a}, \mathrm{b}), 20 \mu \mathrm{m}(\mathrm{g}, \mathrm{h})$. 